\title{
Direct manipulations of cartographic space for expressive cartographies
}

\author{
Nick Lally ${ }^{\text {a }}$ \\ ${ }^{a}$ Department of Geography, University of Kentucky, nicklally@uky.edu
}

Keywords: art, space, speculative cartography, non-euclidean, tools

\begin{abstract}
:
Creative engagements with cartography often benefit from fluid and dynamic understandings of space. Hand drawn maps, like those used in mental mapping projects, and art that uses torn, collaged, and sculpted print maps break with the rigid confines of mathematically projected space. Geographic information systems (GIS), however, largely confine users to a narrow Euclidean conception of projected space. For those who wish to incorporate digitized geographic information into their projects, GIS often becomes a necessity, which also limits the ability to engage that geographic information with more fluid and expressive understandings of space. In this work, I explore how GIS and geographic information might be opened to more expressive forms of direct manipulation, including stretching, sculpting, tearing, and expanding.
\end{abstract}

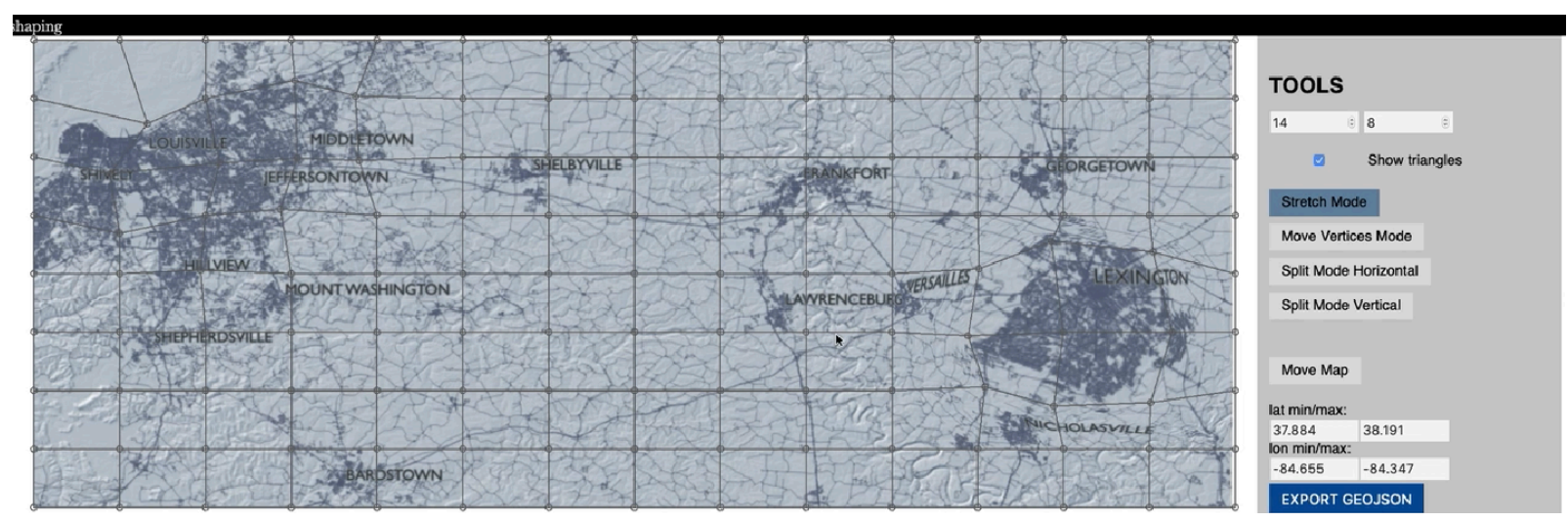

Figure 1. Screenshot of the shaping interface showing the stretching of cartographic space

In this talk, I present shaping, ${ }^{l}$ a browser-based application that I created, which facilitates direct manipulations of cartographic space. The three main functions of shaping are:

1. stretching space

2. tearing space

3. algorithmic expansions of space

These functions work by overlaying a user-defined grid over the map, which can then be directly manipulated with the mouse (see Figure 1). Users can stretch the gridded space by manipulating vertices, create horizontal or vertical tears by clicking on vertices, and algorithmically expand areas of the map by click and dragging at any point on the map. shaping functions much like image editing software and aims to be similarly intuitive, fast, and direct. But unlike image editing software, shaping offers workflows to connect the results of its manipulations to QGIS. Functions 1 and 3 in shaping can be deployed to manipulate raster and vector layers in QGIS. This means that labels, points, and lines do not appear stretched in QGIS, instead they are reconfigured in similar ways as a projection (the manipulations offered by shaping can be conceptualized as a form of projection). Function 2, the tearing of space, is currently offered only as a theoretical extension of QGIS, but torn images can be directly exported as images from shaping (see Figure 2).

shaping is offered here as one possibility for filling some of the space between various artistic and expressive engagements with the materiality of maps and the often rigid structure of GIS. The project is guided by the following open questions: What creative expressions of cartographic visualization become possible when space is no longer a fixed and unchangeable entity? And what tools can we build to facilitate other creative engagements with space?

\footnotetext{
${ }^{1}$ The code for the project can be downloaded and modified at https://github.com/nicklally/shaping and a demo version of the software can be explored at https://nicklally.github.io/shaping/
} 


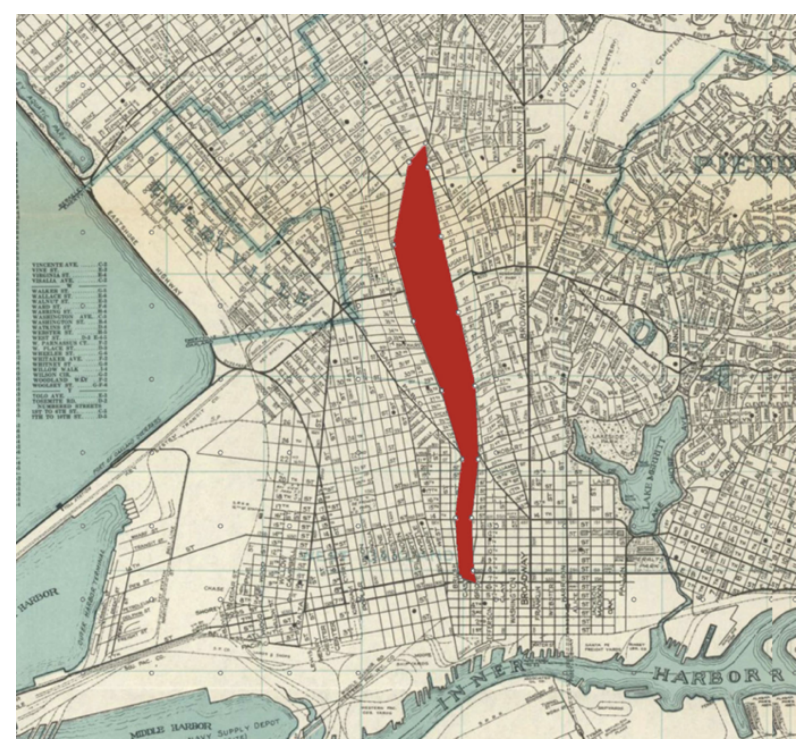

Figure 2. Screenshot of the shaping interface showing the tearing of cartographic space. Here, the I-980 highway tears apart previously adjacent neighborhoods in Oakland, California. 